\title{
Reporte de Peridiniopsis borgei Lemmermann (Dinophyceae) en aguas continentales de Perú
}

\author{
Record of Peridiniopsis borgei Lemmermann (Dinophyceae) in Peruvian inland waters
}

\section{Enrique Ascencio ${ }^{1 *}$, Leonardo Mendoza-Carbajal ${ }^{2}$, Maribel Baylón ${ }^{3}$, Patricio Rivera ${ }^{1}$, Aleksander F. Krakhmalnyi ${ }^{4}$, Fabiola Cruces ${ }^{1}$ \& Roberto Urrutia ${ }^{5,6}$}

\author{
1Departamento de Botánica, Facultad de Ciencias Naturales y Oceanográficas, Universidad de Concepción, Casilla 160-C, Concepción, Chile. \\ 2Departamento de Limnología, Museo de Historia Natural, Universidad Nacional Mayor de San Marcos, Apartado 14-0434, Lima 14, Perú. \\ ${ }^{3}$ Facultad de Ciencias Biológicas, Universidad Nacional Mayor de San Marcos, Apartado 11-0058, Lima 11, Perú. \\ ${ }^{4}$ Institute for Evolutionary Ecology, National Academy of Sciences of Ukraine, 37 Acad. Lebedev Str., Kiev 03143, Ukraine. \\ ${ }_{5}^{5}$ Departamento de Sistemas Acuáticos, Facultad de Ciencias Ambientales \& Centro EULA-Chile, Universidad de Concepción, Casilla 160-C, Concepción, Chile. \\ ${ }^{6}$ Centro de Recursos Hídricos para la Agricultura y la Minería (CRHIAM), Victoria 1295, Barrio Universitario, Concepción, Chile. \\ *E-mail: eaascenc@gmail.com
}

\begin{abstract}
We reported the presence of Peridiniopsis borgei Lemmermann in La Encantada lake (Province of Huaura) and studied the specimens using optical and scanning electron microscopical techniques. The population of $P$. borgei showed a variation in the number and degree of development of the marginal ridges. This is the first illustrated report of the species in Perú.
\end{abstract}

El género Peridiniopsis Lemmermann tradicionalmente se diferencia de Peridinium Ehrenberg por poseer una fórmula tabular generalizada (3-5)'-(0-1)a-(6-7)"-5"'-2"'" (Bourrelly 1968a, Carty 2014, Moestrup \& Calado 2018) y en función de múltiples evidencias morfológicas y genéticas es señalado como un complejo plurigénerico que requiere una profunda revisión crítica e integral (Boltovskoy 1999, Logares et al. 2007, Gottschling et al. 2017, Moestrup \& Calado 2018). Peridiniopsis presenta una amplia distribución mundial (Krakhmalny et al. 2000, Zhang et al. 2012, Carty 2014, Moestrup \& Calado 2018). En América del Sur los estudios taxonómicos enfocados en los dinoflagelados de ecosistemas acuáticos continentales son limitados (e.g., Boltovskoy 1973, 1975, 1999, 2003, Ascencio et al. 2015, 2018, Samanez 2015, Cavalcante et al. 2017) y concretamente los reportes de especies de Peridiniopsis son muy escasos (Meyer et al. 1997, Boltovskoy 1999, Cavalcante et al. 2017).

Hasta el presente, en Perú el género Peridiniopsis está representado por dos especies: Peridiniopsis cunningtonii

Open Access Journal

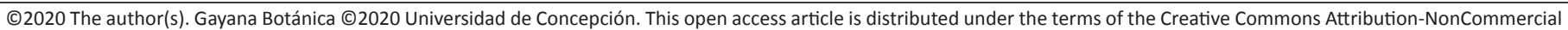
4.0 International License which permits any noncommercial use, distribution, and reproduction, as long as you give appropriate credit to the original author(s) and the source.
Lemmernann reportado en la represa Poechos, un sistema eutrófico subtropical ubicado en el norte del país (López et al. 2017) y Peridiniopsis borgei Lemmermann mencionado en un listado florístico, pero sin una ilustración que permita corroborar su identidad especifica (Hegewald et al. 1980).

Durante un programa de investigación de la microflora algal presente en el humedal laguna La Encantada (Región Lima, Perú) se encontraron tecas de Peridiniopsis borgei. Por ende, el objetivo de la presente comunicación es proporcionar el primer reporte ilustrado de $P$. borgei en Perú y entregar algunos antecedentes ecológicos de la especie.

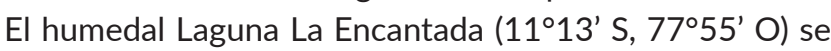
ubica a 135 m.s.n.m, en el distrito de Santa María (Provincia de Huaura, Región Lima) a $6 \mathrm{~km}$ al sureste de la ciudad de Huacho. Este humedal está situado entre las cuencas de los ríos Huaura y Chancay, y está inserto dentro de la ecorregión desierto cálido subtropical sensu Britto (2017), caracterizado por presentar un clima cálido con precipitaciones menores a 200 mm/anuales. El humedal está conformado por un espejo 
de agua principal (Laguna La Encantada) rodeado parcialmente por vegetación acuática y plantas halófitas en los suelos salinizados (especialmente Typha dominguensis Pers., Distichlis spicata (L.) Greene y Cyperus laevigatus L.) y por charcas pequeñas originadas por las filtraciones de napas freáticas (Municipalidad Distrital de Santa María 2011). La Laguna La Encantada tiene aproximadamente una superficie de 35 hectáreas y un perímetro de 2600 m. Alrededor de su cuenca se desarrollan actividades turísticas, agrícolas y un sector de ella está destinada a la acuicultura pesquera de la tilapia (Oreochromis sp.) mediante el sistema de jaulas flotantes. La laguna se ha definido como un sistema eutrofizado y salobre. El cuerpo de agua presenta rangos de temperaturas anuales que oscilan entre 17,5 y $28,5^{\circ} \mathrm{C}$, transparencia del disco Sechi entre 20 y $40 \mathrm{~cm}, \mathrm{pH}$ que fluctúa entre 7,5 y 8,5, oxígeno disuelto entre 1,433 y $1,900 \mathrm{mg} / \mathrm{L}$, Nitritos entre 0,01 y 0,02 $\mathrm{mg} / \mathrm{L}$ y amonio entre 0,2 y $1,0 \mathrm{mg} / \mathrm{L}$ (Benites-Requena et al. 2014).

Las muestras de fitoplancton fueron colectadas durante los meses de abril, agosto y octubre del 2012 con una red de plancton de $20 \mu \mathrm{m}$ de abertura de malla y fijadas con formaldehído al $4 \%$. Además, se obtuvieron las variables ambientales medidas in situ mediante una sonda multiparámetro HANNAH. Las observaciones de los ejemplares del dinoflagelado se realizaron mediante la metodología descrita por Boltovskoy (1995) en un microscopio óptico Leica DM 750 con cámara ICC50 (Facultad de Ciencias Biológicas, Universidad Nacional Mayor de San Marcos) y las observaciones con el microscopio electrónico de barrido (MEB) se realizaron con un microscopio Jeol JSM - 6380 LVY del Centro de Espectroscopia y Microscopia electrónica de la Universidad de Concepción (CESMI-UDEC, Chile).

\section{DESCRIPCIÓN DE LA ESPECIE}

Peridiniopsis borgei Lemmermann, Archiv för Botanik 2(2): 134, pl. I, figs. 1-5. 1904.

Sinónimos: Peridinium borgei (Lemmermann) Lemmermann, Kryptogamenflora der Mark Brandenburg, Algen I: 671. 1910. Glenodinium borgei (Lemmermann) J. Schiller, Dinoflagellatae (Peridineae) 10(3)(2)(1): 112, figs. 108 a-e. 1935.

Los ejemplares de Peridiniopsis borgei encontrados presentaron forma ovalada o esférico-cónica, con notables variaciones en el número y desarrollo de las crestas marginales (Figs. 1a-c). Se observaron individuos con ausencia de crestas apicales y antapicales (Fig. 1a), ejemplares presentaron crestas antapicales con crestas muy rudimentaria o ausentes (Fig. 1b) y también se observaron tecas con cresta marginal entre la sutura 2'/4' y antapicales bien desarrolladas (Fig. 1c). Las tecas presentaron una longitud 27,0-43,0 $\mu \mathrm{m}(38,68 \pm 3,35$ $\mu \mathrm{m})$, longitud total (con crestas marginales incluidas) 37,8 -
48,8 $\mu \mathrm{m}(46,04 \pm 2,94 \mu \mathrm{m})$, transdiámetro 22,8-33,3 $\mu \mathrm{m}$ $(28,39 \pm 2,88 \mu \mathrm{m})$ y un espesor $21,8-27,8 \mu \mathrm{m}(24,54 \pm 2,04$ $\mu \mathrm{m})$. Los ejemplares estudiados presentaron una fórmula tabular: Po, x? , 4' (=3'+1a), 6", 5'", 2'"', 6C, 5S. La superficie de las placas está ornamentada por un reticulado que presenta un poro en cada retículo y en los ejemplares con bandas intercalares desarrolladas se observó que estas se encuentran cubiertas por estrías transversales gruesas. El área apical está conformada por el complejo del poro apical (CPA) (Figs. 1a-e). En la epiteca se observó una placa hexagonal que es interpretada como la tercera apical ( $\left.3^{\prime}\right)$, ya que está conectada con el CPA mediante una sutura corta entre las placas 2'/4'. En esta sutura se observaron crestas marginales conformadas por las aletas de las placas contiguas ( $2^{\prime}$ y $\left.4^{\prime}\right)$ que presentaron distinto grado de desarrollo (Figs. 1a-c, 1e-f), incluso ausente en algunos ejemplares (Fig. 1a). La hipoteca está constituida por 7 placas (cinco placas postcingulares y dos placas antapicales) y entre ambas placas antapicales se observó en algunos ejemplares una cresta marginal de tamaño variable (Figs. 1b-c, 1d-f). El cingulum es descendente, excavado y está conformado por 6 placas de ornamentación menos marcada (Figs. 1e-f). El sulcus es profundo y se ensancha hacia atrás, pero no se extiende hasta el antápice (1f), en el borde derecho se observó una aleta sulcal de gran tamaño y ornamentada (Fig. 1d). La sulcal derecha (Sd) es alargada en sentido longitudinal y presenta en su borde derecho una aleta ornamentada de gránulos (Fig. 1d).

Los rangos de las variables ambientales en los meses analizados fueron: la temperatura superficial del agua de 18,9$22,2^{\circ} \mathrm{C}, \mathrm{pH}$ de $8,4-9,9$, la conductividad de 4,6-5,6 mS/cm y una salinidad media de 4,3 ppm. Peridiniopsis borgei presentó una abundancia relativa moderada y la comunidad algal de la laguna La Encantada estuvo dominada por cianobacterias de los géneros Microcystis Kützing, Planktolyngbya Anagnostidis \& Komárek y Coelemoron H.F. Buell y por diatomeas de los géneros Chaetoceros Ehrenberg y Nitzschia Hassall (L. Mendoza-Carbajal, observaciones personales).

\section{DISCUSIÓN Y CONCLUSIONES GENERALES}

Los ejemplares analizados en nuestro estudio presentan la formula tabular general conocida para la especie. Al analizar los diversos estudios taxonómicos e ilustraciones disponibles en la literatura se infiere que $P$. borgei es una especie muy variable en cuanto al número y tamaño de las crestas marginales, forma celular y grado de desarrollo de la ornamentación superficial. La variación en la forma celular en vista ventral varía desde ejemplares redondeados (Ling et al. 1989: figs. 26-27, 85., Zhang et al. 2012: figs. 2a-b., Krakhlmalny 2014: fig. 1a), esférico-cónicas (nuestras figuras 1b, 1d) a tecas redondeadas con la epiteca de forma piramidal 
(nuestros ejemplares: Figs. 1a, e., Lindemann 1923: figs. 1-2. Bourrelly 1968b: figs. 1-2,5., Pollingher \& Hickel 1991: fig. 2g., Calado \& Moestrup 2002: fig. 1) e individuos ovalados con un notorio cuello apical (Entz 1926: fig. 2). La especie también tiene variación en el número y desarrollo de las crestas marginales, se ha observado ejemplares sin crestas apicales ni antapicales, pero con aletas sulcales en ambos bordes laterales del sulcus (Ling et al. 1989: figs. 85-86, Gätz \& Schager 1990: figs. 1, 3, Calado \& Moestrup 2002: figs. 2-4) o con aletas sulcales rudimentarias (Calado \& Moestrup 2002: fig. 5) y ejemplares con escaso desarrollo de las crestas apicales y antapicales, pero una aleta sulcal derecha destacada (nuestros ejemplares: Figs. 1e-f).

Peridiniopsis borgei fue propuesto artificialmente en conjunto con otras cuatro especies (Peridiniopsis amazonica B. Meyer, Peridiniopsis cristata (Balech) Bourrelly, Peridiniopsis kulczynskii (Wołoszyńska) Bourrelly y Peridiniospsis sphaeroidea Bourrelly) en el "grupo 1 o grupo de P. borgei" sensu Moestrup $\&$ Calado (2018). Este grupo se caracteriza por una epiteca con 10 placas + CPA (Po, x, 3', 1a, 6", también interpretado como Po, x, 4', 6"). Sin embargo, entre todas las especies asignadas al "grupo 1", Peridiniopsis borgei sólo tiene afinidad morfológica, la misma constitución tecal e idéntica tabulación con $P$. cristata. Esta última especie es considerada endémica del atolón de Clipperton, donde se han encontrado dos variedades: P. cristata var. cristata (Balech 1961, Ricard \& Bourrelly 1982, Couté et al. 2012) y Peridiniopsis cristata var. tubulifera Couté, Perrette \& Chomérat que se caracteriza por la ausencia de ornamentación superficial (Couté et al. 2012). Una tercera variedad, Peridiniopsis cristata var. boliviensis Iltis \& Couté, sólo ha sido señalada en el lago Poopó y lago Titicaca en Bolivia (Iltis \& Couté 1984, Cadima et al. 2005). Boltovskoy
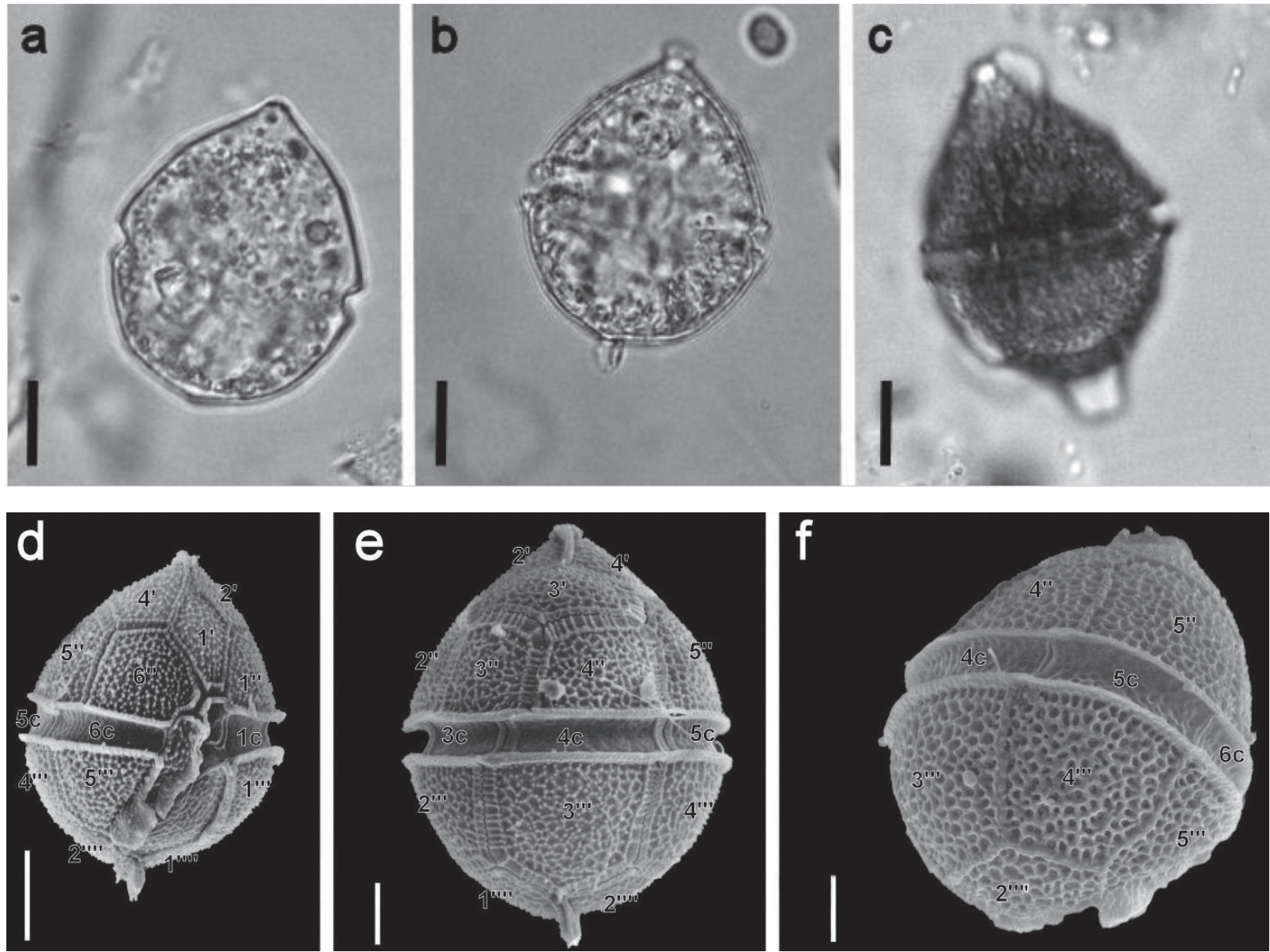

Figura 1. Peridiniopsis borgei de la Laguna La Encantada, Perú. A-C: variación morfológica de P. borgei en microscopía fotónica. D-F: imágenes en MEB de P. borgei. D: vista ventral. E: vista dorsal. F: Vista lateral derecha. Escalas: A-D=10 $\mu \mathrm{m}, \mathrm{E}-\mathrm{F}=5 \mu \mathrm{m} / \mathrm{Peridiniopsis}$ borgei from La Encantada lake (Perú). A-C: morphological variation of P. borgei in light microscopy. D-F: images SEM of P. borgei. D: ventral view. $E$ : dorsal view. F: right side view. Scale bars: $A-D=10 \mu \mathrm{m}, E-F=5 \mu \mathrm{m}$. 
(1999) estudió distintas poblaciones de P. borgei en lagos argentinos, donde encontró una amplia variación en el número de crestas marginales, grado de desarrollo de la ornamentación superficial, forma de los individuos y la constitución tecal, interpretando a Peridiniopsis cristata (incluidas las variedades conocidas hasta ese momento: $P$. cristata var. cristata y $P$. cristata var. boliviensis) como variaciones morfológicas, incluidas dentro del rango de variación de Peridiniopsis borgei y concluye que el amplio espectro de variabilidad fenotípica de la especie podría estar relacionada a las condiciones ecológicas (especialmente la salinidad). Sin embargo, se requiere un estudio polifásico, incluyendo análisis de la ultraestructura y estudios moleculares de distintas poblaciones que permitan corroborar la propuesta de Boltovskoy (1999).

Peridiniopsis borgei tiene amplia distribución geográfica en ecosistemas de agua dulce y salobre, siendo mencionada en distintas localidades de Europa (Lemmermann 1904, Entz 1926, Lefèvre 1932, Gätz \& Schagerl 1990, Calado \& Moestrup 2002, Krakhmalny 2014), Asia (Pollingher \& Hickel 1991, Krakhmalny et al. 2000, Zhang et al. 2012), Norteamérica (Carty 2014) y Oceanía (Ling et al. 1989, Ling \& Tyler 2000). En América del Sur se le encontró en distintos cuerpos de aguas salobres de Argentina (Boltovskoy 1999) y en la laguna Piuray (Andes Peruanos, 3400 m.s.n.m.), pero este reporte carece de ilustraciones de la especie mencionada (Hegewal et al. 1980). Por ende, el presente estudio confirma la presencia de $P$. borgei en el Perú, ampliando su distribución en el país y contribuye al conocimiento de la diversidad biológica de los humedales costeros del centro de Perú, donde los estudios en torno a las microalgas son muy escasos y centrados en las cianobacterias y algas verdes (v.g., Montoya 1984, Aguilar et al. 2004, Mendoza-Carbajal 2016).

Debido que P. borgei ha originado eventos de proliferaciones masivas en Argentina (Boltovskoy 1999), Estados Unidos (Whitford \& Schumacher 1984) y Finlandia (Lindholm \& Öhman 1995) se sugiere realizar estudios ecológicos detallados que permitan conocer la dinámica poblacional de $P$. borgei en la laguna La Encantada.

\section{AGRADECIMIENTOS}

Agradecemos a la Municipalidad Distrital de Santa María (Huacho) por el apoyo logístico durante la actividad de campo y la asistencia técnica del personal de CESMI-UDEC (Chile) en el procesamiento del material para microscopia electrónica. También agradecemos a los Drs. A. Boltovskoy, A.J. Calado, T. Lindholm y D. Seamer por el envío de literatura de difícil acceso. Investigación parcialmente financiada por VRIDUDEC y CRHIAM Conicyt/Fondap/15130015.

\section{REFERENCIAS}

Aguilar, C.P., González, M., Cifuentes, A.S., Silva, M. 2004. Growth and accumulation of total carotenoids in two strains of Dunaliella salina Teod. (Chlorophyceae) from the Northern and Central coast of Perú. Journal of the Chilean Chemical Society 49(1): 69-74. http://dx.doi.org/10.4067/S071797072004000100012

Ascencio, E., Rivera, P., Cruces, F. 2015. Morfología de Peridiniopsis elpatiewskyi (Ostenfeld) Bourrelly (Dinophyceae) encontrada por primera vez en aguas continentales de Chile. Gayana Botánica 72(1): 42-46. http://dx.doi. org/10.4067/S0717-66432015000100006

Ascencio, E., Rivera, P., Cruces, F., Vila, I. 2018. Estudio morfológico de Naiadinium polonicum (Dinophyceae) reportado por primera vez en aguas continentales de Chile. Gayana Botánica 75(1): 438-446. http://dx.doi. org/10.4067/S0717-66432018000100438

Balech, E. 1961. Glenodinium cristatum sp. nov. (Dinoflagellata). Neotropica 7: 47-51.

Benites-Requena, E.S., Calderon de los Rios, H.D., Cotos-Durán, D.E., Salvador-Zurita, M. 2014. Factores abióticos y su influencia en la eutrofización estacional en las aguas de la laguna La Encantada, año 2013. Universidad Nacional José Faustino Sánchez Carrión, Lima. 13 pp.

Boltovskoy, A. 1973. Peridinium gatunense Nygaard. Estructura y estereoultraestructura tecal (Dinoflagellida). Physis B 32(85): 331-344.

Boltovskoy, A. 1975. Estructura y estereoultraestructura tecal de dinoflagelados. II. Peridinium cinctum (Müller) Ehrenberg. Physis B 34(89): 73-84.

Boltovskoy,A. 1995. Taxonomíaymorfología de los dinoflagelados: Métodos de trabajo. En: Alveal, K., Ferrario, M.E., Oliveira, E.C., Sar, E. (Eds.) Manual de métodos ficológicos: 55-82. Universidad de Concepción, Concepción, Chile.

Boltovskoy, A. 1999. Contribución al conocimiento de los dinoflagelados de la república Argentina. Tesis doctoral. Facultad de Ciencias Naturales y Museo, Universidad Nacional de La Plata. La Plata, Argentina. 148 pp.

Boltovskoy, A. 2003. Peridinium willei (Dinophyceae) y sus formas: ecología y distribución en la Argentina. Boletín de la Sociedad Argentina de Botánica 38(1-2): 175 -184.

Bourrelly, P. 1968a. Notes sur les Péridiniens d'eau douce. Protistologica 4(1): 5-16.

Bourrelly, P. 1968b. Note sur Peridiniopsis borgei Lemm. Phykos 7: 1-2.

Britto, B. 2017. Actualización de las ecorregiones terrestres de Perú propuestas en el libro rojo de plantas endémicas del Perú. Gayana Botánica 74(1): 15-29. http://dx.doi. org/10.4067/S0717-66432017005000318

Cadima, F.M., Fernández, T.E., López, Z.L. 2005. Algas de Bolivia con énfasis en el fitoplancton: importancia, ecología, aplicaciones y distribución de géneros. Centro de Ecología Difusión Simón I. Patiño, Santa Cruz de la Sierra. 378 pp.

Calado, A.J., Moestrup, Ø. 2002. Ultrastructural study of the 
species of Peridiniopsis, Peridiniopsis borgei (Dinophyceae), with special reference to the peduncle and flagellar apparatus. Phycologia 41(6): 567-584. https://doi. org/10.2216/i0031-8884-41-6-567.1

Carty, S. 2014. Freshwater dinoflagellates of North America. Cornell University Press, Ithaca. 260 pp. https://www. jstor.org/stable/10.7591/j.ctt5hh1q4

Cavalcante, K.P., Craveiro, S.C., Calado, A.J., Ludwig, T.A.V., Cardoso, L.S. 2017. Diversity of freshwater dinoflagellates in the State of Paraná, southern Brazil, with taxonomic and distributional notes. Fottea 17(2): 240-263. https:// doi.org/10.5507/fot.2016.026

Couté, A., Perrette, C., Chomérat, N. 2012. Three Dinophyceae from Clipperton Island lagoon (Eastern Pacific Ocean), including a description of Peridiniopsis cristata var. tubulifera var. nov. Botanica Marina 55: 59-71. https://doi. org/10.1515/bot-2011-121

Entz, G. 1926. Beiträge zur kenntnis der Peridineen. I. Zur morphologie und biologie von Peridinium Borgei Lemmermann. Archiv für Protistenkunde 56: 397-446.

Gätz, N., Schagerl, M. 1990. Peridiniopsis borgei Lemm.- eine seltene Dinophyceae in ziegelteichen in Wien und Niederösterreich. Lauterbornia H 4: 24-29.

Gottschling, M., Kretschmann, J., Žerdoner-Čalasan, A. 2017. Description of Peridiniopsidaceae, fam. nov. (Peridiniales, Dinophyceae). Phytotaxa 299(2): 293-296. https://doi. org/10.11646/phytotaxa.299.2.16

Hegewald, E., Schnepf, E., Aldave, A. 1980. Investigation on the lakes of Peru and their phytoplankton. 5. The algae of Laguna Piuray and Laguna Huaypo, Cuzco, with special reference to Franceia, Oocystis and Scenedesmus. Archiv für Hydrobiologie 56/4 (Algological Studies 25): 387-420.

Iltis, A., Couté, A. 1984. Péridiniales (Algae, Pyrrhophyta) de Bolivie. Revue d'Hydrobiologie Tropicale 17(4): 279-286.

Krakhmalny, A.F. 2014. New species of dinoflagellata (Dinoflagellate) for Ukraine. In: Nature of Polessia: research and conservation, pp. 330-333. Rivnenska Daukarnia, Kiev, Ukraine.

Krakhmalny, A.F., Wasser, S.P., Nevo, E. 2000. Dinophyta. In: Nevo, E., Wasser, S.P. (Eds.) Biodiversity of Cyanoprocaryotes, algae and fungi of Israel: Cyanoprocaryotes and algae of continental Israel: 177-183. A.R.A. Gantner Verlag K.G. Ruggell, Germany.

Lefèvre, M. 1932. Monographie des espèces d'eau douce du genre Peridinium Ehrb. Archives de Botanique 2(5): 1-216.

Lemmermann, E. 1904. Das planton schwedischer gewässer. Arkiv för Botanik 2(2): 1-209.

Lindemann, E. 1923. Eine Entwicklungshemmug bei Peridinium borgei und ihre Folgen. Archiv für Protistenkunde 46: 378-382.

Lindholm, T., Öhman, P. 1995. Occurrence of Bloom-forming and potentially harmful phytoplankton in a Åland archipelago in the summer of 1993. Memoranda Societatis pro Fauna et Flora Fennica 71(1): 10-18.

Ling, H.U., Tyler, P.A. 2000. Australian freshwater algae (exclusive of Diatoms). Bibliotheca Phycologica 105: 1-643.

Ling, H.U., Croome, R.L., Tyler, P.A. 1989. Freshwater dinoflagellates of Tasmania, a survey of taxonomy and distribution. British Phycological Journal 24: 111-129. https://doi.org/10.1080/000716189.00650111

Logares, R., Shalchian-Tabrizi, K., Boltovskoy, A., Rengefors, K. 2007. Extensive dinoflagellate phylogenies indicate infrequent marine-freshwater transitions. Molecular Phylogenetics and Evolution 45: 887-903. http://doi. org/10.1016/j.ympev.2007.08.005

López, J., Yucra, H., Bárcena, V., Saavedra, R. 2017. Morfología y taxonomía de dinoflagelados tecados (Peridiniales, Peridiniaceae) en la represa Tahuin (Oro, Ecuador) y Poechos (Sullana, Perú). The Biologist 15(1): 99-108. http://dx.doi.org/10.24039/rtb2017151146

Mendoza-Carbajal, L. 2016. El género Sphaerocavum y dominancia de S. brasiliense y Microcystis wesenbergii (Microcystaceae, Cyanophyceae) en la floración algal de la laguna Huacachina, Perú. Revista Peruana de Biología 23(1): 5360. http://dx.doi.org/10.15381/rpb.v23i1.11835

Meyer, B., Rai, H., Cronberg, G. 1997. The thecal structure of Peridiniopsis amazonica spec. nov. (Dinophyceae), a new cyst-producing freshwater dinoflagellate from Amazonian floodplain lakes. Nova Hedwigia 65 (1-4): 365-375.

Moestrup, Ø., Calado, A.J. 2018. Dinophyceae. In: Büdel, B., Gärtner, G., Krienitz, L., Schagerl, M. (Eds.) Süßwasserflora von Mitteleuropa 6: 1-561. Springer Spektrum, Berlin, Germany. https://doi.org/10.1007/978-3-662-56269-7

Montoya, H. 1984. Algas de la Laguna de Villa (Lima): Cyanophyta, Chlorophyta y Chrysophyta (Xanthophyphyceae). Boletin de Lima 31(6): 75-89.

Municipalidad Distrital de Santa María. 2011. Plan de desarrollo concertado del distrito de Santa María 2011-2021. Santa María, Perú. 138 pp.

Pollingher, U., Hickel, B. 1991. Dinoflagellate associations in a subtropical lake (Lake Kinneret, Israel). Archiv für Hydrobiologie 120(3): 267-285.

Ricard, M., Bourrelly, P. 1982. Quelques algues microscopiques du lagon de l'Atoll de Clipperton (Pacifique Tropical Nord). Cryptogamie Algologie 3: 25-31.

Samanez, I. 2015. Primer reporte de Lophodinium polylophum (Daday)Lemmermann1910(Dinophyceae:Lophodiniaceae) en Perú. Revista Peruana de Biología 22(1): 109-112. https://doi.org/10.15381/rpb.v22i1.11128

Whitford, L.A., Schumacher, G.J. 1984. A manual of fresh-water algae. Sparks Press.

Zhang, Q., Liu, G.X., Hu, Z.Y. 2012. Study on freshwater genus Peridiniopsis (Dinophyta) from China. Acta Hydrobiologica Sinica 36(4): 751-764. https://doi.org/100.3724/ SP.J.1035.2012.00751

Received: 06.10.2019

Accepted: 19.03.2020 